Meta

Journal des tradlucteurs

Translators' Journal

\title{
Problèmes de traduction dans le domaine de la poésie chantée
}

\section{Suzanne de Grandmont}

Volume 23, numéro 1, mars 1978

La traduction poétique

URI : https://id.erudit.org/iderudit/002959ar

DOI : https://doi.org/10.7202/002959ar

Aller au sommaire du numéro

Éditeur(s)

Les Presses de l'Université de Montréal

ISSN

0026-0452 (imprimé)

1492-1421 (numérique)

Découvrir la revue

Citer cet article

de Grandmont, S. (1978). Problèmes de traduction dans le domaine de la poésie chantée. Meta, 23(1), 97-108. https://doi.org/10.7202/002959ar d'utilisation que vous pouvez consulter en ligne.

https://apropos.erudit.org/fr/usagers/politique-dutilisation/ 


\section{Problèmes de traduction dans le domaine de la poésie chantée}

\section{Nous vivions tout...}

Ces mots tirés de la très belle chanson de Jacques Prévert et de Joseph Kosma, les Feuilles mortes, combien de fois les avons-nous fredonnés durant les semaines que nous avons consacrées à traduire The Fantasticks, la comédie musicale de l'auteur Tom Jones, et du compositeur Harvey Schmidt ${ }^{1}$ ! Des trois mots de Prévert, nous fîmes une sorte de syntagme plaisant pour rappeler à l'ordre les traducteurs et leur indiquer avec humour : danger, syncope, mauvaise coupure, «que faites-vous de l'accent tonique $»$ ? Les trois mots fatidiques nous avaient été inspirés par le récital que Montand donna, à Paris, au Théâtre de l'Étoile et qu'il enregistra sur disque Odéon. Avec enthousiasme et une certaine inconscience, le bel Yves a chanté :

\section{Nous vivions tout... \\ Les deux ensemble.}

"Le père Chevalier, Maurice de son prénom, ne s'est pas laissé prendre, lui. Fidèle à la partition, et avec le discernement qui le caractérisait, il a chanté :

Et... nous vivions...

Tous deux ensemble.

Deux interprétations d'un même texte. Pour faire fausse route, il ne suffisait à Montand que d'oublier une blanche... et de faire une pause à contretemps.

Lorsque le poète Daniel Slote me proposa de rédiger un article sur la traduction poétique, il fut catégorique : « Il ne faut surtout pas écrire un article anecdotique. »Ce n'est pas par espièglerie ni par esprit d'insubordination que j'ai choisi de commencer mon exposé par une anecdote, c'est que ce petit fait «peut éclairer le dessous des choses».

Connaître le dessous des choses, lire entre les lignes, peser les mots, accorder la priorité au sens, faire fi de la littéralité, rechercher des équivalences tant dynamiques que contextuelles ne sont-ils pas les «must» (n'en déplaise à M. Beaudry) de l'opération traduisante ? Nous savons tous, hélas ! que traduire de la prose n'est jamais chose facile. Mais, lorsqu'un texte poétique est

1. En 1964, Jean Gascon confia à Eloi de Grandmont la traduction de : * The Fantas ticks». Eloi s'adjoignit deux collaborateurs, sa femme et Jean Rafa. La contribution de Rafa fut précieuse. Il nous a aidés et conseillés dans l'art délicat des couplets $\$$ 
mis en musique, l'œuvre du traducteur devient travail de bénédictin car, à toutes les difficultés inhérentes à la traduction, s'ajoutent les exigences de la cadence, de la mesure, du tempo et de l'accent tonique. Il faut remettre en question la marge de liberté du traducteur. Puis, dans le feu de l'action, lorsque les problèmes semblent insurmontables il importe de se rappeler, ne serait-ce que pour se rassurer, que Goethe a écrit : «Si j'étais encore assez jeune et assez osé, je violerais à dessein toutes les lois de fantaisie, j'userais des allitérations, des assonances, des fausses rimes et de tout ce qui me semblerait commode... ${ }^{2} \gg$

Avant de parler de la marge de liberté que l'on peut souhaiter dans une traduction poétique, une question se pose. Peut-on qualifier le livret de Tom Jones d'œuvre poétique? Oui, si l'on reconnaît avec J. Charpier et P. Seghers qu'il n'y a pas de la poésie une définition mais cent, et souvent contradictoires. Oui, si l'on juge comme Larousse que le livret versifié d'un opéra ou d'une pièce lyrique est un poème. Oui, si, selon la même source, l'œuvre « offre et suggère à l'imagination poétique une riche et merveilleuse matière ».

Or, le livret des Fantasticks est une ouvre d'une naïveté belle et déconcertante, comme une toile du célèbre Douanier; le rêve est son univers et sa fantaisie poétique évoque le Grand Meaulnes. Rares sont les poèmes à formes fixes où le nombre de vers, le croisement des rimes, l'ordre général soient fixés par des règles. Le vers libre moderne et les poèmes en prose sont à l'honneur.

Tom Jones s'est surtout attaché à la poésie rythmique dans laquelle la mesure se compose d'un certain nombre de syllabes, sans tenir compte de la quantité. $\mathrm{Il}$ a surtout fait appel à la musique du vers, préférant souvent la mesure à la rime. Les accents toniques et la coupe du vers mettent en valeur la cadence. Cette cadence si chère à Malherbe, à Boileau et à notre grand poète québécois, Alfred Desrochers.

Pour traduire et adapter la comédie musicale The Fantasticks nous savions, avant même d'ouvrir le livret, que nous aurions à surmonter des problèmes importants. Nous savions aussi que les chansons d'une comédie musicale font partie intégrante du dialogue et de l'action dramatique et qu'il ne fallait pas les traiter comme les morceaux de bravoure d'un opéra où d'une opérette où les grands airs servent à mettre en valeur les acrobaties vocales des artistes, désireux de conquérir l'auditoire en poussant un contre-ré étourdissant. Nous savions aussi que cette comédie de mœurs accordait une large place au rire et à l'humour. Or, l'humour est parfois intraduisible. Et l'on n'a pas encore, à ce jour, trouvé de recette infaillible pour faire rire. «C'est une étrange entreprise que celle de faire rire les honnêtes gens », disait Molière.

Comment obtenir l'équivalence dynamique? C'était le premier impératif qui nous préoccupait: que notre traduction produise sur les spectateurs du Théâtre du Nouveau Monde le même effet que l'œuvre originale avait eu sur le public du Sullivan Street Playhouse.

2. Remy de Gourmont; Esthétique de la langue française, p. 145. 
La lecture du livret nous apprit que l'œuvre d'Edmond Rostand, les Romanesques, avait inspiré la création de la comédie musicale The Fantasticks ${ }^{3}$. En découvrant la chose, grande fut notre consternation. Eloi, perplexe, se posa la question : «Faudra-t-il que j'écrive du Rostand?» Il ajouta : « Je n'en avais pas le goût. Et puis, si on faisait cela, ce serait pour en rire. Non.» Dans le programme du TNM il s'explique ainsi : «Après avoir lu et relu la pièce américaine j'allai sur la pointe des pieds, à la Municipale, lire les Romanesques. Disons tout de suite que cette comédie est une petite chose gracieuse; désuète, poudreuse, adorable qui n'a rien à voir avec The Fantasticks... » Tom Jones reprend l'argument de Rostand, pas un mot de plus. En refermant le livret, la première question qui nous vint à l'esprit : devons-nous traduire le titre littéralement ou le changer ? Après avoir longuement réfléchi, Éloi décida de garder le titre original. «Ce mot : Fantasticks n'est pas français; il n'est pas anglais non plus. C'est un mot fabriqué dans le but de souligner la fantaisie nouvelle et la grâce touchante de l'œuvre de Tom Jones et de Harvey Schmidt. »Alea jacta est! Nous avions d'un commun accord réglé le premier problème de notre traduction.

Connaître à fond les personnages de la comédie, les cerner de près pour les faire dialoguer «selon leur genre », selon leur niveau de langue, telle fut la deuxième étape. La distribution compte huit personnages. Nous les présentons accompagnés d'un court profil psychologique afin que le lecteur comprenne les difficultés qui ont surgi quand il a fallu rendre le niveau de langue de chaque rôle.

\section{HUIT PERSONNAGES EN QUÊTE DE TRADUCTEURS}

The Mute

Traduction libre : le Muet! Comme son nom l'indique si bien, il ne posait aucun problème aux traducteurs.

The Narrator, El Gallo

Le narrateur : El Gallo conservera son nom sonore et exotique car c'est un citoyen de 1'Univers. Il tient tout à la fois du picaro, du saltimbanque et du philosophe. Son langage est châtié et fleuri.

The Girl, Luisa

La jeune fille, nous l'avons prénommée Louise. Elle a seize ans, imbue de littérature et de mythologie, elle s'y réfère souvent. Mythomane, elle prétend que «chaque année pour mon anniversaire la lune devient rouge». Elle se croit princesse et affirme que son nom devrait être espagnol. Snob, elle avoue "personne ne peut ressentir ce que je ressens et avoir un père qui se nomme Joseph-Adéodat-Hildège-Gourmelon »! Elle parle une langue belle, harmonieuse et riche en allusions littéraires.

The Boy, Matt

Marc : le jeune blanc-bec par excellence, joue les intellectuels, les érudits, les savants. «J'ai étudié la biologie, je suis déjà entré dans un laboratoire et j'ai disséqué des violettes. M Mythomane, par contagion peut-être,

3. Ironie du sort! L'édition du livret The Fantasticks, publiée par Drama Book Shop, New York, 1964, n'a pas corrigé l'erreur qui s'était glissée dans le livret publié en 1959. Edmond Rostand, à l'américaine est devenu « Edmund \$: Le père de l'Aiglon a dû se réjouir, là-haut, quand le programme Showcard a orthographié correctement son prénom. 
The Girl's Father, Bellamy

The Boy's Father, Hucklebee

The old Actor, Henry

The Man who dies, Mortimer il se dit fou comme Hamlet, déchaîné comme le roi Lear. Poète à ses heures, il écrit des sonnets à la lune. Sa langue est celle d'un jeune rhétoricien éloquent.

Le père de la jeune fille... La tentation était grande de l'appeler Bonami. Nous avons résisté en l'appelant Gourmelon parce qu'il est jardinier amateur, passionné de kumquats. Son nouveau nom évoque ses plates-bandes. Sa langue sent le terroir.

Le père du jeune homme est également amateur de jardinage. Il parle, lui aussi, une langue familière. Parce qu'il est fort préoccupé par le problème de la soif de ses plantes, nous l'avons dénommé Robineau. Petite allusion à la soif proverbiale des robineux ${ }^{4}$. Les deux pères sont deux clowns. Leur pitrerie cache une intention didactique. Il ne s'agit pas, pour eux, de faire la leçon aux rois comme dans les moralités mais d'indiquer, aux parents et aux enfants du $\mathrm{XX}^{\mathrm{e}}$ siècle, la profondeur du fossé qui les sépare. Nous analyserons "leur idéologie » dans la chanson «Plant a radish».

Firmin Pot : le vieil acteur shakespearien a des trous de mémoire. Atteint de sénilité, après quarante ans de métier, il fausse et tronque toutes les citations. Selon le cas, sa langue est châtiée ou vulgaire.

Parce que depuis quarante ans, il sait savamment mourir sur scène il aura nom Mortier. Sa langue est familière. Firmin Pot et Mortier sont des personnages surréalistes, des clowns servant de repoussoir à l'intrigant El Gallo. Par la drôlerie de leurs gestes, par leurs bredouillements et par leurs discours farfelus ils sont les héritiers directs des comiques de Molière.

Les pauvres pères bernés, Robineau et Gourmelon désirent unir leur progéniture par les liens du mariage; ils ignorent, cependant, que Louise et Marc sont déjà amoureux. Pour réussir leur doux projet ils construisent un mur pour séparer leurs jardins contigus et ils jouent les Montaigus et les Capulets. Lorsqu'ils constatent que les enfants sont amoureux ils s'imaginent qu'ils ont réussi. Comment les deux ennemis vont-ils, alors, se réconcilier? Leur imagination fertile invente un scénario rocambolesque. Les vieux prient El Gallo d'organiser, avec des comparses, l'enlèvement de Louise en échange d'une généreuse rémunération. Le rapt est réglê comme une scène de Molière : bastonnade, coups d'épée, Indiens à plumes. Marc, héroïque, se porte à la défense de Louise et triomphe, tel que prévu. La première partie de la comédie se déroule à la faveur de la nuit.

$\mathrm{Au}$ début de la seconde partie, le lendemain, le soleil ardent dissipe les tếnèbres et le romanesque. Louise et Marc se querellent et se quittent, le complot des pères semble compromis. Après maintes péripéties, les deux enfants qui ont souffert mais qui ont vieilli en sagesse s'avouent leur amour tandis que l'ami El Gallo philosophe sur l'étrange paradoxe :

4. N. du T, - A Montréal, on appelle nos clochards des « robineux ». 
Who understands why spring is born out of winter's laboring pain ?

Qui peut comprendre pourquoi le printemps naît des chagrins de l'hiver?

Il y avait onze chansons à traduire dans la comédie musicale : six dans la première partie et cinq dans la seconde. Il serait trop long de les analyser toutes. Nous nous contenterons d'en donner la liste et de marquer d'un astérisque celles sur lesquelles nous nous attarderons.

Première partie

Try to remember*

Much more*

Metaphor

Never say no*

It depends on what you pay*

Soon it's gonna rain

Try to remember c'est la chanson-thème de la comédie. Ecrite, sur une musique à trois temps - valse lente - elle crée l'ambiance qui nous introduit dans le monde idyllique des «fantasticks». El Gallo chante les trois strophes. Le refrain, tout simple : "Follow, follow, follow, follow», est repris par la troupe. Dire que traduire les couplets était facile serait une litote. Comment concilier les exigences de la fidélité, l'importance de la cadence et la place de l'accent tonique? Roger Lesourd, partition en main, clavier au bout des doigts rejetait nos ébauches en nous sérénadant : «Nous vivions tout... 》 Malgré tout, l'humour reprenait toujours le dessus, notre équipe travaillant dans la joie. La difficulté qui nous paraissait insurmontable, c'était l'accent tonique portant sur l'impératif "try », verbe d'une syllabe. Nous avons compté et recompté toutes les syllabes des trois strophes, nous avons cherché à traduire fidèlement «try ». Nous avons écouté le disque, à en périr, car, au début, nous n'avions pas la partition. La solution ne s'offrit pas spontanément. Un matin, à l'aube, Éloi trouva. En utilisant le verbe « chercher » à l'impératif, l'accent tonique porterait sur la première syllabe. Victoire! Le poète pouvait donner libre cours à sa créativité. Nous proposons, à l'analyse, la première strophe. Les accents toniques sont en italique.

TRY TO REMEMBER

Try to remember the kind of September

When life was slow and $o h$, so mellow.

Try to remember the kind of September

When grass was green and grain was yellow.

Try to remember the kind of September

When you were a tender and callow fellow.

\section{CHERCHE DANS TON CEUR}

Cherche dans ton cœur septembre où le bonheur

Etait si doux et l'âme rose.

Cherche dans ton coeur septembre et le bonheur...

Partout sur terre les fleurs se posent.

Cherche dans ton coeur septembre où le bonheur

Avait pour toi changé l'odeur des choses.

Cherche dans ton cour et s'il le veut ton cour, 
Try to remember, and if you remem-

Là, suis-nous. Suis-nous, suis-nous.

ber,

Then follow, follow, follow.

La bataille, mais non la guerre, contre l'accent tonique terminée, l'équivalence dynamique demeura notre principal souci. Comment évoquer la nostalgie de la fin de l'été, du mois de septembre sans recourir à la modulation ?

When grass was green and grain was yellow.

Partout sur terre les fleurs se posent.

La saison des blés d'or et des moissons n'annonce-t-elle pas, comme la chute des fleurs, la fin de l'été - septembre ? Autre modulation importante, le verbe « remember». Même si nous sommes d'un pays qui a devise « Je me souviens», les exigences du rythme ne nous permettaient pas de traduire servilement ce verbe. Le poète a créé la mémoire du cœur.

Tous les soirs, après chaque représentation, le public quittait le théâtre en chantant "Cherche dans ton cœur», comme le public newyorkais fredonnait : «Try to remember ». Ce phénomène peut-il s'appeler «équivalence dynamique $\gg$ ? Oui, de l'avis des connaisseurs.

Dans la chanson Much more, Louise nous confie ses chimères. Elle commence par un récitatif sur une musique à quatre temps, accompagnant la première strophe et le début de la deuxième. Mais sur « I'd like to dance till two o'clock», subitement le rythme change, s'accélère, devient très syncopé c'est une musique de jazz, "style béguine », rythmée sur des contretemps et des notes de valeurs irrégulières. Les lettres en italique indiquent les accents toniques. Le double trait vertical indique : attaque syncopée pour le chanteur mais temps fort donné à l'accompagnement. L'emploi fréquent de cette syncope est une des caractéristiques du jazz.

\section{MUCH MORE}

I'd like to swim in a clear blue stream Where the water is icy cold; Then go to town in a golden gown, And have my fortune told.

Just once.

Just once.

Just once before I'm old.

I'd like to be - not evil,

But a little wordly wise.

Tó be the kind of girl designed

To be kissed upon the eyes.

I'd like to dance till || two o'clock,

Or sometimes dance till dawn,

Or if the band could stand it,

Just go on and on and on!

Just once,

Just once,

Before the chance is gone!

\section{UN JOUR}

Je veux nager dans une onde pure, Vêtue de soie et de velours

Pour connaître la grande aventure

Au pays de l'amour

Un jour,

Un jour,

Et pourquoi pas demain?

Je serai une dame,

De galants chevaliers

Pour me montrer toute leur flamme Se jetteront à mes pieds.

Un jour, un jour je $\|$ danserai

Et je m'étourdirai.

Un air léger

Jouera

Pour moi

Pour moi

Jouera

Un jour

Un jour

Un jour

Je danserai l'amour! 
Il est évident que nous avons rejeté la traduction littérale pour lui préférer la modulation libre, en changeant de point de vue, d'éclairage et de catégorie de pensée. Nous croyons cependant avoir respecté le personnage de Louise qui rêve de luxe et de grandeur. C'est ainsi que «Then go to town in a golden gown », est devenu «Vêtue de soie et de velours». Une dame au sens véritable du mot, a, la plupart du temps, acquis une certaine sagesse - "Wordly wise», tandis que la petite fille rêveuse et snob qui prétend être une princesse, cherchera de galants chevaliers qui ne l'embrasseront que «Upon the eyes». Cela va de soi.

Adapter Never say no, le duo des deux pères, fut cauchemardesque parce qu'il y avait alternance de rythme. Les deux premières strophes sont écrites sur une musique à quatre temps, tandis que la mesure de la troisième est à deux temps. Il en est ainsi pour toute la chanson. Le rythme binaire, c'est le rythme de la marche. Il est donc scandé. Pour respecter la mesure que ne fit-on pas! Comme les enfants qui apprennent l'arithmétique nous comptions les syllabes sur nos doigts. Les résultats étaient minables. Le poète trouva la solution : "Les chansons populaires ont recours à la synérèse quand les syllabes muettes gênent pour la mesure. Pourquoi ne pas en faire autant?»Never say no, cette chanson humoristique est écrite dans le style de la poésie populaire qui, comme on le sait, est le pays de la licence, de toutes les licences...

Au nom de la mesure, Jones a dû également surmonter des obstacles semblables aux nôtres puisque dans la première strophe il utilise la contraction : «a mule's got to bray ». Il recourt au même procédé dans la deuxième strophe ainsi que dans la troisième. La chanson raillait les théories discutables du docteur Spock. Elle amusa le public parce que les deux pères pédagogues nous livraient leurs théories sur l'éducation des enfants non seulement en chantant mais en dansant sur un rythme de tango argentin.

NEVER SAY NO

Ohhhhhhhh -

Dog's got to bark; a Mule's got to bray

Soldiers must fight and preachers must pray,

And children, I guess, must get their own way

The minute that you say no.

Why did the kids pour jam on the cat?

Raspberry jam all over the cat?

Why should the kids do something like that,

When all that we said was «no»?

\section{Hucklebee}

My son was once afraid to swim ;

The water made him wince.

Until I said he must'nt swim;

S'been swimmin' ever since!
FAUT PAS DIRE NON

$\mathrm{Oh} ! \ldots$

Le chien doit japper, la mul' doit braire.

Monsieur l'curé doit fair' des prières.

L'enfant, je crois, veut fair' le contraire.

Sitôt qu'on lui a dit «non».

Pourquoi les jeun's barbouill'n-ilst les chats

De confiture ou de chocolat?

Pourquoi les jeun's font-ils ces cho'slà

Quand on leur a just' dit «non».

Robineau

Mon fils ne voulait pas nager,

N'aimant pas la marine!

L'lui ai défendu d'se baigner,

Y sort plus d'la piscine!

Y sort plus d'la piscine! 
Jusqu'ici, nous avons choisi les chansons qui nous permettaient de traiter des problèmes de la cadence, du niveau de langue, de l'équivalence dynamique, etc. La chanson It depends on what you pay nous offre l'occasion d'effleurer le sujet de la rime et celui de l'adaptation en fonction de la métalinguistique.

IT DEPENDS ON WHAT YOU PAY

Rape !

R-a-a-a-pe !

Raa-aa-aa-pe!

A pretty rape.

Such a pretty rape!

We've the obvious open schoolboy rape,

With little mandolins and perhaps a cape,

The rape by coach ; it's little in request.

The rape by day; but the rape by night is best.

Just try to see it,

And you will soon agree, senors,

Why invite regret,

When you can get the sort of rape

You'll never ever forget!
TOUT DÉPEND DU PRIX QU'ON PAIE

Rap...pt!

Ra...a...a...a...pt!

Ra...aa...aa...aa...apt !

Un beau p'tit rapt.

Un beau p'tit rapt.

Un si beau p'tit rapt!

On a le petit rapt pour écolier

Avec guitare dans un paso doble.

Rapt en carrosse, mais c'est démodé.

Le rapt en plein jour, mais c'est mieux la nuit!

L'occasion passe.

Faut la saisir.

Faut la retenir.

On est très aptes.

Quand on les capte,

On les adapte,

On a tous les genres de rapts !

Ce trio ou le chant du rapt, chanté par El Gallo et les pères, débute par une psalmodie rappelant l'appel du muezzin. Après «Un si beau p'tit rapt!» le narrateur adopte la pose du danseur de flamenco et attaque la deuxième strophe sur le rythme endiablé d'un paso doble. Voilà pourquoi paso doble rime avec écolier et démodé, ce ne sont pas des rimes riches mais des assonances qui auraient peut-être réjoui Goethe. Qui sait?

«Démodé » traduit «It's little in request ». Tom Jones n'a pas donné de rime à « it». Nous avons pris une licence semblable, au dernier vers de la deuxième strophe, parce que nous avons préféré le sens à la rime.

$\mathrm{Au}$ milieu de la même chanson, El Gallo, vantant la variété de rapts qu'il peut offrir, s'explique ainsi :

The spectacular rape, with costumes ordered from the East.

Requires rehearsal - and takes a dozen men at least.

A couple of singers

And a string quartet.

A major production - requires a set.

Pour «interpréter * cette chanson, nous avons dû compenser « en rétablissant sur un autre point de l'énoncé la nuance qui n'a pas été rendue au même endroit que dans l'original ${ }^{5} »$. Il a fallu que notre traduction soit oblique :

5. J.P. Vinay et J. Darbelnet, Stylistique comparée du français et de l'anglais. 
L'enlèvement au sérail, (évoque «spectacular rape 》 et "major produc-

Avec des harpes et des violons, (évoque «string quartet».)

tion $\gg$.)

Pour un meilleur travail,

On love des costumes chez Ponton!

Pour rendre « with costumes ordered from the East » dont la traduction littérale n'aurait eu, au Québec, aucune résonance, notre modulation nous a conduits chez Ponton. Joseph Ponton Inc., le costumier qui depuis 1865 habille les gens de théâtre, les fervents de masquarades et les pères Noël. Nommer Ponton, c'était un procédé métalinguistique de faire comprendre au public que la comédie musicale avait été adaptée pour Montréal.

Le quatuor This plum is too ripe chanté par les pères et les enfants, au début de la seconde partie de la comédie, annonce que rien ne va plus : les amoureux se quittent et les pères déplorent l'échec de leur petit scénario. Pour nous, aussi, rien n'allait plus. Fallait-il renoncer à adapter cette chanson, à cause de son rythme? La partition indique «Swing 4 with a jazz beat ». Quand on sait que le démon de la liberté domine le jazz, le rythme « jazzy », convulsif de cette chanson était d'une belle diablerie, mais devant les difficultés qu'elle présentait, nous nous sommes demandé ce que nous faisions dans notre galère. En écoutant le disque nous avons tenté de trouver les temps forts, de les bien noter en divisant les syllabes de la façon suivante :

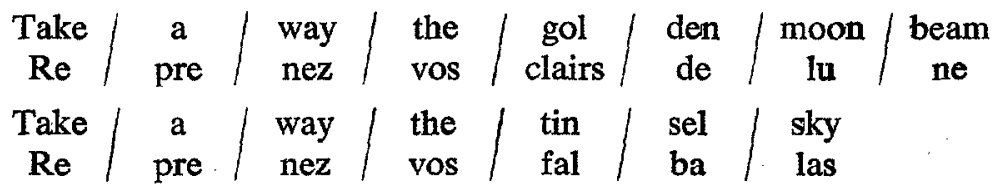

Peine perdue, nous avions bien compté le nombre de syllabes, mais où étaient les accents forts ? Roger Lesourd vint à notre rescousse et grâce à la partition, nous avons pu «re-créer » la chanson, en lui donnant l'interprétation qui suit. (Les temps forts sont en italique, le double trait vertical indique également un temps fort donné à l'accompagnement.)

THIS PLUM IS TOO RIPE

II Take away the golden moonbeam.

II Take away the tinsel sky. II

II What at night seems oh so scenic,

|| May be cynic by and by. II
REMBALLEZ TOUT ÇA

II Reprenez vos clairs de lune.

|| Remballez vos falbalas. ||

II Dans la nuit, tout ce qui brille

\|I Au grand jour, n'a plus d'éclat.

Le duo des pères Plant a radish est écrit sur une musique à deux temps. Les musiciens modernes qualifient ce rythme de «style Broadway», celui du «soft shoe». Après Try to remember c'est la chanson la plus importante de l'œuvre, celle qui a la plus grande signification. Les pères utilisent un langage familier lorsqu'ils nous exposent avec verve, dans une langue savoureuse et drue, les problèmes de la paternité. Les vieux viennent de constater que leur stratagème, pour forcer Louise et Marc à se marier a échoué. Ils se consolent avec humour en comparant les enfants aux légumes. Les légumes sont les grands 
vainqueurs parce que, avec les plantes potagères, on sait où l'on va. Mais avec ses propres rejetons, c'est différent car
While with progeny
It's hodge podgenee
La progéniture
C'est une aventure

Hodge-podge, le terme tire son origine du vocabulaire culinaire. Webster le définit ainsi : (alter. of hotchpot) 1. - a heterogeneous mixture often of incongruous and ill-suited elements : Mixture, Medley. 2. - Hotchpotch. Harrap nous renvoie uniquement à Hotchpotch qu'il traduit : $1 . \mathrm{cu}:$ Hochepot, salmigondis, galimafrée... «vegetable hochepot», macédoine de légumes. 2. F. mélange confus ; méli-mélo, macédoine, salmigondis, salade russe, ripopée (de vieilles théories, etc.) Par curiosité nous avons cherché hochepot dans les dictionnaires français. Il n'existe ni dans le Grand Robert ni dans le Petit Robert. Lexis définit hochepot : \& ragoût de viandes diverses, cuites avec des marrons ou des navets. » Le Grand Larousse nous propose : «sorte de pot-au-feu avec porc, bœuf, mouton et légumes divers $\gg . .$. ? Allez donc vous y retrouver! Pour ne pas s'y perdre, il faut oublier les dictionnaires et moduler. Moduler et cogiter car la chanson était longue et citait d'autres légumes pour lesquels il fallait trouver des équivalents, tout en respectant le tempo.

Voici comment nous avons traité les deux premières strophes. Vous constaterez que les synérèses furent indispensables à Tom Jones autant qu'à nous.

PLANT A RADISH

Plant a radish ;

Get a radish.

Never any doubt

That's why $I$ love vegetables;

You know what you're about!

Plant a turnip; get a turnip,

Maybe you'll get two.

That's why I love vegetables;

You know that they'll come through!

\section{J'AI DES RADIS}

J'ai des radis.

Ils ont grandi.

J'les ai récoltés.

Les légum's, ça n' tromp' jamais.

On récolt' c'qu'on a s'mé!

Moi, mes navets,

Ils sont parfaits.

C'est un vrai succès.

Les légumes, ça tromp' jamais ;

On récol' c'qu'on a s'mé!

Le duo They were you chante la réconciliation de Marc et de Louise, sur un rythme de valse lente. Ce n'est pas sans émotion que je vous offre l'œuvre du poète, en version intégrale, et la livre à vos commentaires. La chanson a été «re-créée » de façon si admirable, elle est si touchante que je n'ose pas, cette fois, la disséquer comme Marc le ferait de ses violettes.

THEY WERE YOU

\section{Matt}

When the moon was young,

When the month was May

When the stage was hung for my holiday,

I saw shining lights

But I never knew

They were you

They were you
C'ÉTAIT NOUS

Marc

Quand le clair de lune

De ce mois de mai

Pour nous faire la fête,

S'est illuminé,

Je n'ai pas compris

Que ce paradis

C'était toi 
They were you.

\section{Luisa}

When the dance was done,

When I went my way,

When I tried to find rainbows far away,

All the lovely lights seemed to fade from view

They were you

They were you

They were you.

\section{Both}

Without you near me,

I can't see.

When you're near me

Wonderful things come to be.

Ev'ry secret prayer,

Every fancy free,

Ev'ry thing I dared for both you and me,

All my wildest dreams multiplied by two,

They were you

They were you

They were you.

Matt

They were you

\section{Luisa}

They were you

\section{Both}

They were you.
C'était toi

C'était toi!

\section{Louise}

Nous avons dansé,

Puis je t'ai quitté.

Brusquement la lune

Pour moi s'est cachée.

Je n'ai pas compris

Cette sombre nuit

C'était toi

C'était toi

C'était toi !

\section{Ensemble}

Sans toi

Près de moi

Je ne vois

Les merveilles

$\mathrm{Ni}$ de la terre ni du ciel.

Tous les voux du cour

Même les plus fous,

Tout ce grand bonheur

Que j'attends pour nous

Me semblent faciles

Et voici pourquoi :

C'était toi

C'était toi

C'était toi !

Marc

C'était toi

Louise

C'était toi

\section{Ensemble}

C'était nous!

Je désire remercier sincèrement les personnes qui ont collaboré à la préparation de mon article :

- Mademoiselle Colombe Pelletier, la grande pianiste qui m'a gracieusement prêté la partition : The Fantasticks, et qui m'a consacré une soirée, pour revoir en détail, et la partition et les livrets ;

- Monsieur Victor Lamarche, Madame Suzanne Lamarche, Mademoiselle Marie-Claire Delcenserie, trois amis professeurs qui ont enregistré sur bobines la musique et les paroles des disques utiles à mon analyse; 
- Monsicur Guy Archambault, professeur à l'École supérieure de musique Vincent-d'Indy qui m'a aidée à identifier avec précision les différents rythmes de danse de la comédie;

- Mademoiselle Agathe Proulx du Centre de musique canadienne dont les conseils techniques furent précieux;

- Monsieur François Cardin, discothécaire à C.K.V.L. qui a retrouvé les disques anciens dont j'avais besoin.

Suzanne de Grandmont 